\title{
Laser Thin Film Patterning for Rapid Prototyping and Customised Production of Flexible Electronics Devices
}

\author{
Nicola Bellini ${ }^{1}$, Riccardo Geremia ${ }^{1}$, Shane Norval ${ }^{2}$, Guillaume Fichet ${ }^{2}$ and Dimitris Karnakis*1 \\ ${ }^{1}$ Oxford Lasers Ltd, Unit 8 Moorbrook Park, Didcot, OX11 7HP United Kingdom \\ ${ }^{*}$ E-mail: dimitris.karnakis@oxfordlasers.com \\ ${ }^{2}$ FlexEnable Ltd, 34 Cambridge Science Park, Cambridge, CB4 OFX United Kingdom
}

\begin{abstract}
We present ultrafast laser processing (source/drain transistor patterning and via drilling for interconnections) for customizable fabrication of flexible display organic thin film transistor (OTFT) backplanes. The process is aimed as an alternative to photolithographic patterning which impedes much needed prototyping efforts currently in this market segment due to its high associated costs and lengthy development cycles. We demonstrate that ultrafast (picosecond and femtosecond) diode-pumped solid-state (DPSS) lasers can be an attractive alternative as a high selective digital fabrication technique, if the correct choice of laser wavelength, pulse duration and incident laser power is made. Optimum process recipes are shown for both (a) source/drain electrode patterning of organic transistor channels and (b) single pulse high density via drilling, by etching a narrow $5 \mu \mathrm{m}$ wide line in a thin metal layer $(80 \mathrm{~nm})$ to fabricate the organic transistor source/drain channel in the former and selective removal of a sub- $\mu$ m thick transparent dielectric layer on top of an ultrathin (80 $\mathrm{nm}$ ) metal layer in the latter case. Both sets of results are correlated with electrical characterization to compare the transistor performance with standard ones obtained by conventional photolithography. These results present a step forward towards a practical and industrially viable implementation of direct-write laser processing for flexible electronics fabrication.
\end{abstract}

DOI: 10.2961/jlmn.2016.03.0019

Keywords: ultrafast laser, picosecond, femtosecond, flexible electronics, display backplane, organic transistors, thermal stress assisted ablation, spatial confinement

\section{Introduction}

Flexible displays require organic thin film transistor (OTFT) backplanes [1], i.e. thin film stacks of metal and transparent polymeric layers forming the on/off pixel switching circuitry. As these layers need to be patterned and interconnected depending on the device design, lithography based batch nanofabrication techniques are typically used (photolithography, nanoimprint lithography, etc.) [2] for high-volume transistor patterning offering high resolution and good edge control. However, lithography cannot be used economically for low or medium volume prototyping runs due to its high associated costs including the design and production of several projection masks or moulds, etc. Contrasting this, laser ablation combined with mechanical servo and optical axes is an attractive alternative being a fully digital direct-write technique [3]. It unlocks unlimited prototyping potential with total design flexibility for patterning layers. Laser ablation can be selective, since the correct choice of laser wavelength, pulse duration and incident laser energy allows for precise removal of only the targeted material with no or very limited detrimental effects on surrounding layers. This becomes extremely useful for patterning layered stacks of several superimposed thin films in the production of organic light emitting diodes (OLED) [4, 5, 6], inorganic copper indium gallium selenide (CIGS) [7, 8, 9], flexible batteries [10], organic photovoltaic solar cells (OPV) [11] or flexible sensors [12].

In this paper, we demonstrate thin film patterning of flexible display transistors utilizing specifically ultrafast diode-pumped solid-state (DPSS) lasers (picosecond and femtosecond). In particular, two transistor patterning processes are investigated and discussed: (a) source/drain (S/D) metal electrode patterning of organic transistor channels and (b) single pulse high density shallow via drilling. The former process consists in cutting a line in a thin metal layer $(80 \mathrm{~nm})$ to fabricate the organic transistor S/D channel before the remaining layers are deposited in the usual manner to complete the thin film transistor stack. The latter consists of single pulse via drilling by selective removal of a sub- $\mu m$ thick transparent dielectric layer on top of an ultrathin $(80 \mathrm{~nm})$ metal layer with the goal of subsequently depositing and connecting two metal layers.

Laser exposure parameters are carefully optimised and tailored for the specific material and task. The effects of laser wavelength and pulse duration are experimentally studied by using picosecond and femtosecond DPSS lasers. Comparison between different lasers will be shown and discussed. In addition, the possibility of reshaping the beam profile on target is also exploited. The laser patterning results are correlated with electrical tests to compare the transistor performance with standard ones obtained by conventional photolithography. These results represent a further step forward for a practical and industrially viable implementation of direct laser processing for the flexible electronics market.

\section{Experimental setup}

The tested thin film stacks consist of (Fig.1) either 


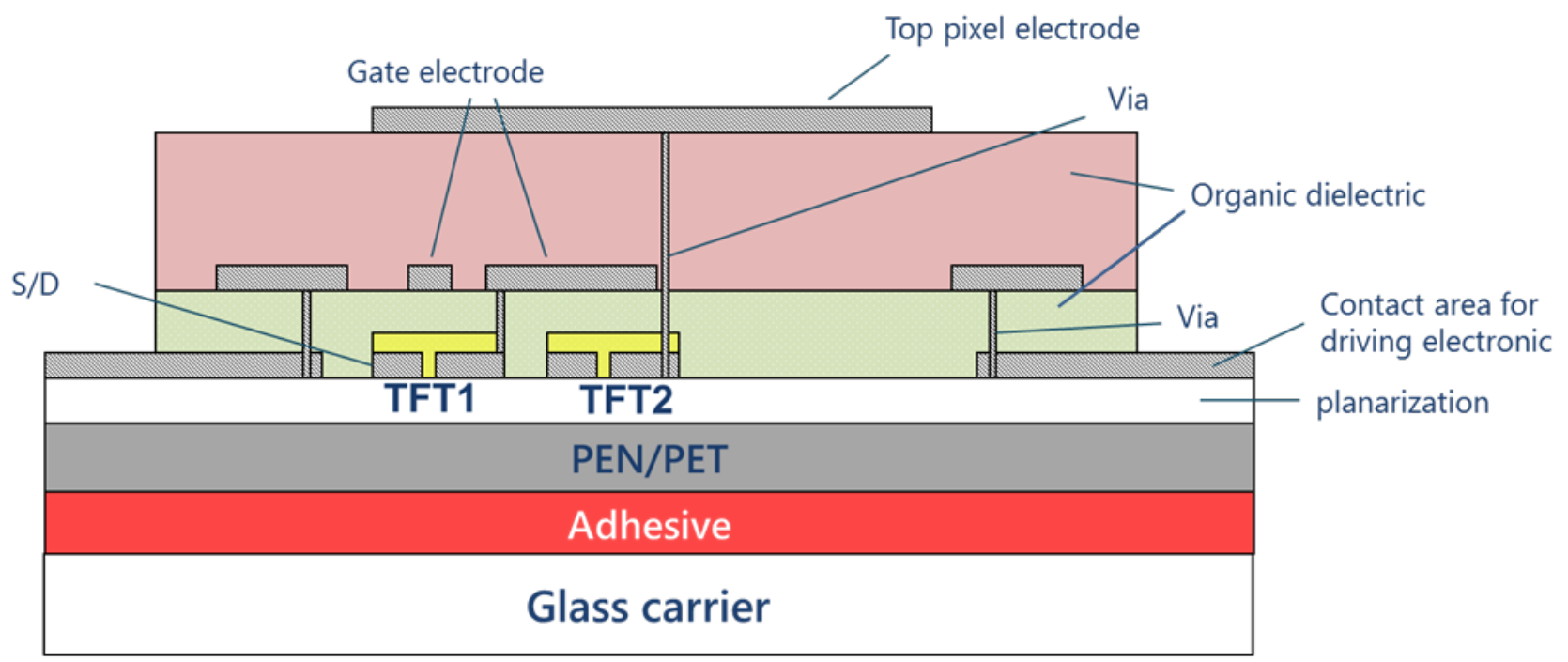

Fig. 1 Backplane stack cross section showing source drain (S/D) metal layer patterned channels for TFT1 and TFT2 transistors (laser process a) and drilled vias (laser process b) through organic dielectric layers

(a) S/D patterning a $80 \mathrm{~nm}$ thick gold-based layer on a 5 $\mu \mathrm{m}$ thick organic planarisation layer or (b) via drilling a $500 \mathrm{~nm}$ thick organic dielectric layer coated on an $80 \mathrm{~nm}$ metallic gold-based layer on top of a $5 \mu \mathrm{m}$ thick organic planarisation layer on flexible polyethylene terephthalate/polyethylene naphthalate (PET/PEN) substrate. The objective is to laser pattern thin metal electrodes cleanly for any given line geometry to form the S/D electrode channel in any bespoke top gate transistor configuration for display backplane production and also to provide vias for interconnection between layers.

Two different laser setups were used. A commercial femtosecond DPSS laser amplifier was used emitting at $1026 \mathrm{~nm}$ pulses of $220 \mathrm{fs}$ duration. The expanded $7.3 \mathrm{~mm}$ beam diameter at $1 / \mathrm{e}^{2}$ was passed through an aperture and then focused with an aberration corrected triplet lens ( $\mathrm{f}=$ $25 \mathrm{~mm}$ ) to an estimated truncated Gaussian beam with diameter of $9.8 \mu \mathrm{m}$ (at $1 / \mathrm{e}^{2}$ ). The dielectric layer is almost totally transparent at $1026 \mathrm{~nm}$. The metal linear absorption coefficient $\alpha$ and reflectivity $\mathrm{R}$ at $1026 \mathrm{~nm}$ and $532 \mathrm{~nm}$ is estimated as $\alpha[1026]=8.1 \times 10^{5} \mathrm{~cm}^{-1}, \mathrm{R}[1026]=97 \%$ and $\alpha[532]=5 \times 10^{5} \mathrm{~cm}^{-1}, \mathrm{R}[532]=68 \%$ respectively from $\mathrm{n}, \mathrm{k}$ optical values provided by the supplier. All layers underneath the metal S/D electrodes were believed transparent at both $1026 \mathrm{~nm}$ and $532 \mathrm{~nm}$.

For S/D patterning a J-series Oxford Lasers micromachining system [13] equipped with a 15 ps duration DPSS laser was used at $532 \mathrm{~nm}$. A similar beam delivery setup was utilised to focus the truncated Gaussian beam to an 8.3 $\mu \mathrm{m}$ diameter spot using a $59 \mathrm{~mm}$ focal length lens. The focused spot was translated across the metal surface using mechanical servo axes to etch a straight line.

Ablation took place in air. In both cases, the pulse energy was varied using a half-waveplate and polariser motorized attenuation setup. The ablated samples were investigated with a high magnification optical microscope or SEM. For electrical characterization of transistor via drilling and transistor performance, FlexEnable used a semiconductor parameter analyser (Keysight 4155B/C).

\section{Results and discussion}

\subsection{Source-Drain electrode patterning}

The single-pulse ablation threshold (average) fluence of the $80 \mathrm{~nm}$ thick gold-based metal layer at $532 \mathrm{~nm}$ (15 ps) is believed to be about F[th] $20-25 \mathrm{~mJ} / \mathrm{cm}^{2}$, much lower than literature reported [14] values for thicker gold films, but in line with our observations. Different incident laser fluences above threshold were used to irradiate the target between $30-40 \mathrm{~mJ} / \mathrm{cm}^{2}$. By translating the focused laser beam on target at different ratios of pulse frequency to scan speed, the local energy input per area (pulse overlap) was varied resulting in different shape channels as shown in Fig. 2. For very low pulse overlap $\mathrm{O}=\Phi \times \mathrm{RR} / \mathrm{s}<1$, where $\Phi$ is the crater diameter on target, $\mathrm{RR}$ is the laser repetition rate and $\mathrm{s}$ is the scan speed, i.e. using a longer laser firing distance than the focused laser spot size, individual ablated craters were produced. By increasing the pulse overlap (reducing the laser firing distance), a continuous etched line begins to form shaped in "connecting arc" serrated edges. Such arcs and their associated sharp joining points are usually undesirable as they can form unwanted electrostatic charge density collection points and disrupt transistor operation.

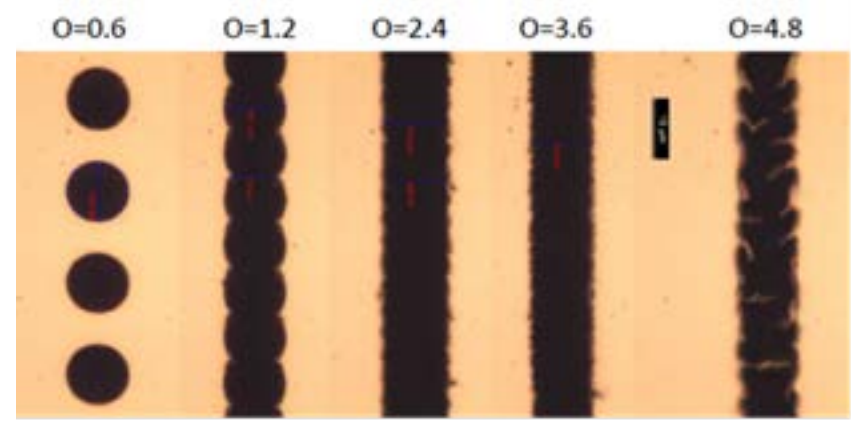

Fig. 2 Optical micrograph of $532 \mathrm{~nm} 15$ ps laser patterned 80 $\mathrm{nm}$ thick gold-based film on polymer at different laser pulse overlap indicated above each line. Incident pulse energy $45 \mathrm{~nJ}$, laser repetition rate $400 \mathrm{~Hz}$. The vertical dark bar denotes $10 \mu \mathrm{m}$. 


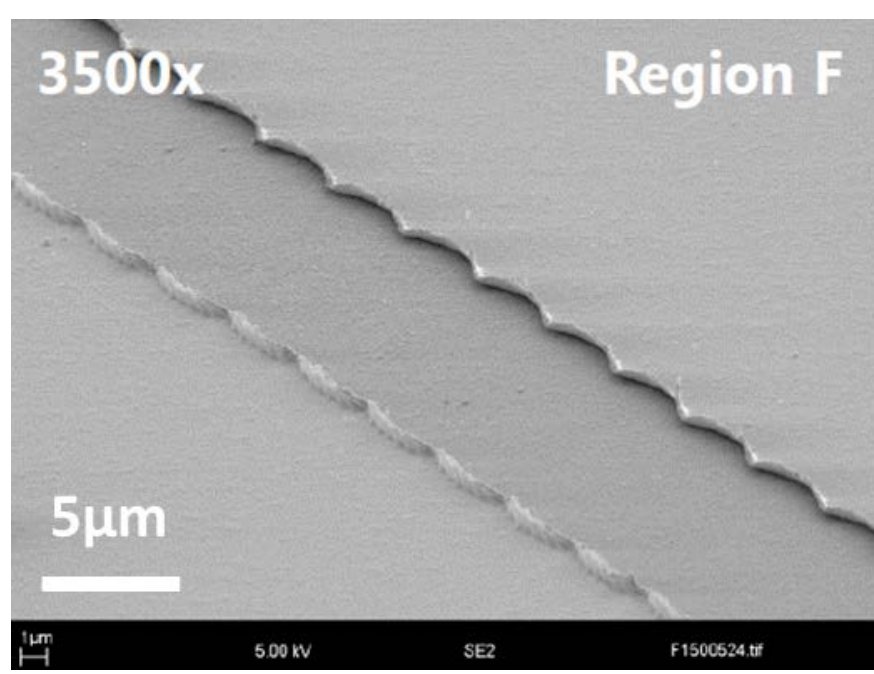

Fig. 3 SEM micrograph of optimum Au patterned using a $532 \mathrm{~nm}$, 15 ps DPSS laser. $60 \mathrm{~nJ}$ per pulse, pulse overlap 3.6 pulses per area, scan speed $1 \mathrm{~mm} / \mathrm{s}$. Bar bottom left denotes $5 \mu \mathrm{m}$.

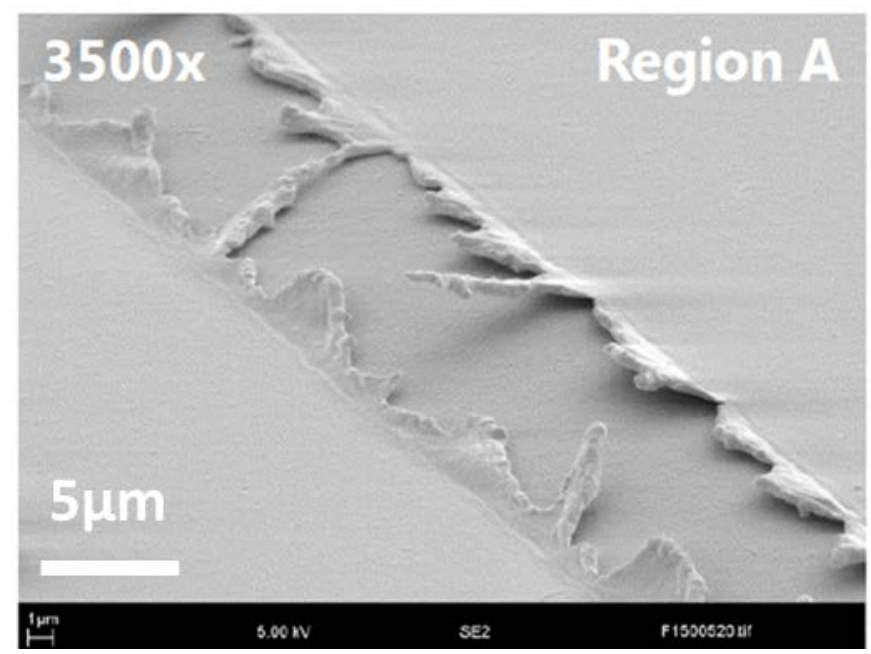

Fig. 4 SEM micrograph of sample in Fig.2 irradiated at different laser conditions: $532 \mathrm{~nm}, 15$ ps DPSS laser, $95 \mathrm{~nJ}$ per pulse, pulse overlap 8 pulses per area, scan speed $0.5 \mathrm{~mm} / \mathrm{s}$. Bar bottom left denotes $5 \mu \mathrm{m}$.

Further increase of pulse overlap creates lines of almost straight edges and an optimum pulse overlap range exists between 2 and 4 pulses per area which results in welldefined, cleanly etched narrow channels where the metal layer is fully removed with, remarkably, no leftover residue and the polymer planarization underlayer remaining intact (Fig.3). Using higher incident pulse overlap from typically four pulses per area and above, results in poor quality etched tracks with highly fractured morphology and irregularly shaped edges. Sharp edge spikes or loosened flakes of metal material raised significantly above the surface are observed with sometimes connecting bridges of nonremoved metal also present (Fig. 4). Even for a very high pulse overlap ranging between 25-250 pulses per area, highly irregular channel edges were observed (not shown here), a rather counterintuitive observation as straight edges are typically to be expected.

For all examined cases, limited evidence of layer melting exists, an observation also supported by the complete lack of redeposited particulate debris around the irradiated zones which is otherwise a common problem in such applications. That is a clear sign of a stress-assisted ablation mechanism [15] at play, where laser-induced thermal stress and shockwaves generated in the absorbing metal film lead to layer fracturing and ejection at low elevated temperatures usually below the melt point. A similar phenomenon has been observed in femtosecond-laser-induced delamination and blister formation of thermal oxide films on silicon and described in ref [16]. In our case, assuming uniform laser heating across the entire $80 \mathrm{~nm}$ metal layer from optical absorption and ballistic electron diffusion, both the thermal and stress relaxation characteristic times at 5 ps and 16 ps respectively, are closely compared to the 15 ps laser pulse duration and so thermal and stress confinement cannot be assumed [17]. Rapid heating proceeds storing sufficient thermoelastic energy which upon release with layer expansion can fracture the metal layer in tension. A simple estimate [18] of the maximum thermal stress experienced in the film is $\sigma=1.2 \mathrm{GPa}$, using $\sigma=\mathrm{A}_{\mathrm{LTE}} \mathrm{E}_{\mathrm{Y}} \Delta \mathrm{T} /$ 2(1-v) with headline values for linear thermal expansion $\mathrm{A}_{\mathrm{LTE}}=1.4 \times 10^{-5} \mathrm{~K}^{-1}$, Young's modulus $\mathrm{E}_{\mathrm{Y}}=79 \mathrm{GPa}$, Poisson ratio $v=0.44$ for cold gold and a temperature rise of about $1300 \mathrm{~K}$ close to the melt point exceeds the gold yield strength of near $220 \mathrm{MPa}$. The exact mechanical properties for the stack layers are not precisely known, but are unlikely to depart too drastically from above estimates.

A possible qualitative explanation for the observed degradation of etched quality with increasing pulse overlap at low irradiating fluences could be as follows: the optical, physicochemical and thermomechanical properties of the repetitively exposed irradiated areas in the film can gradually differ from their original unirradiated values, developing a kind of "memory effect" due to cumulative heating/cooling cycles with a non-homogeneous temperature profile developing across it. Within any one unit of laser irradiated surface area, both highly non-uniform optical absorption and mechanical response can be expected with increasing pulse overlap. Despite the slow $\mathrm{kHz}$ frequency exposure in this case, material changes are possible in between pulses due to plastic deformation and other physical modifications, i.e. material melting and recrystallization causing slight reflectivity changes for each consecutive laser pulse, the film becoming softer, less brittle, acquiring a different residual stress profile with repetitive exposure as its Young's modulus and tensile strength dynamically decrease with increasing temperature. The net result could be that gradually the laser-induced thermal stress experienced locally exceeds the modified material yield strength not only at the track edges, as in the case for single pulse ablation, but at several locations within the irradiated track width and result in highly non-uniform flake fracturing. At the extreme case of very high pulse overlap at low incident fluences, spallation driven ablation could be hindered entirely by a drastic decrease in the thermally-induced stress across the irradiated track length from a transiently modified much lower elasticity modulus. In this case, the adhesion properties of the stack or other pre-existing intrinsic stress from layer deposition could also influence layer removal for such low fluence values.

The apparent laser process window, allowed us to build and test successfully a series of thin film transistors by picosecond laser patterning a $5 \mu \mathrm{m}$ channel length between 
S/D gold-based electrodes. The full transistor construction with gate and other organic semiconductor and dielectric layers and final interconnection circuitry were deposited as per standard recipe at FlexEnable labs. All tested devices exhibited good electrical transistor behavior and could be switched with good reproducibility, satisfactory on/off ratios of $10^{4}$ or over and no disruption of charge injection observed despite the high off currents intrinsic to the structures.

Figure 5 shows typical transistor output curves which allow identifying good charge injection within the linear region (drain source voltage $\mathrm{Vds}=0$ to $-10 \mathrm{~V}$ ) where the performance is linear and passing by the origin indicating a very low contact resistance.

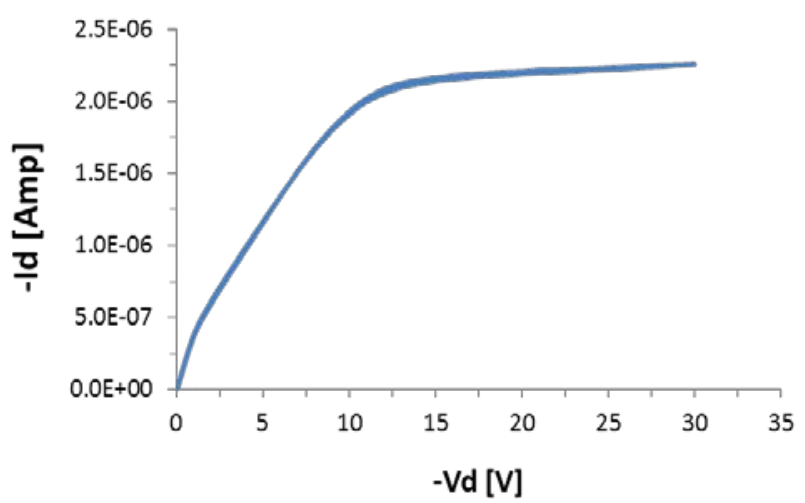

Fig. 5 Output characteristic of laser digitally-defined organic transistors for threshold voltage Vgs $=-15 \mathrm{~V}$.

Digitally-defined channel for organic transistors are conventionally defined by printing [19]. The work carried out by laser ablation is providing superior benefits such as a better accuracy of the patterning, ability to create shortest channel length which enables higher transistor performance and higher process reproducibility. All these factors are critical for a reliable industrial process.

\subsection{Via drilling of dielectric on metal layer}

For selective removal of the $500 \mathrm{~nm}$ thick transparent organic dielectric layer to stop on the $80 \mathrm{~nm}$ metal layer preparing for a subsequent layer interconnection step, picosecond laser trials with second (SHG) and third harmonic generated (THG) upconverted wavelengths were inconclusive. In most cases, the resulting morphology of the ablated dielectric layers appeared like "volcano-shaped dome" craters (Fig.6). The craters were raised near the edge, well above the layer surface and were accompanied by surface cracks, evidence of melting and some scattered redeposited debris. More importantly, a larger diameter area of the metal underlayer, as compared to the dielectric "volcano dome" size, was also etched away. This was highly undesirable, as it made it impossible to establish electrical via connection between the OTFT electrodes by post-process metal evaporation.

In contrast, femtosecond laser trials resulted in a wider process window and selective layer ablation. The single pulse ablation threshold of the metal layer only, in air, was measured as $0.25 \pm 0.04 \mathrm{~J} / \mathrm{cm}^{2}$ using the $220 \mathrm{fs}$ laser pulse. Since the dielectric layer is mostly transparent at $1026 \mathrm{~nm}$, much higher laser fluences were required to selectively

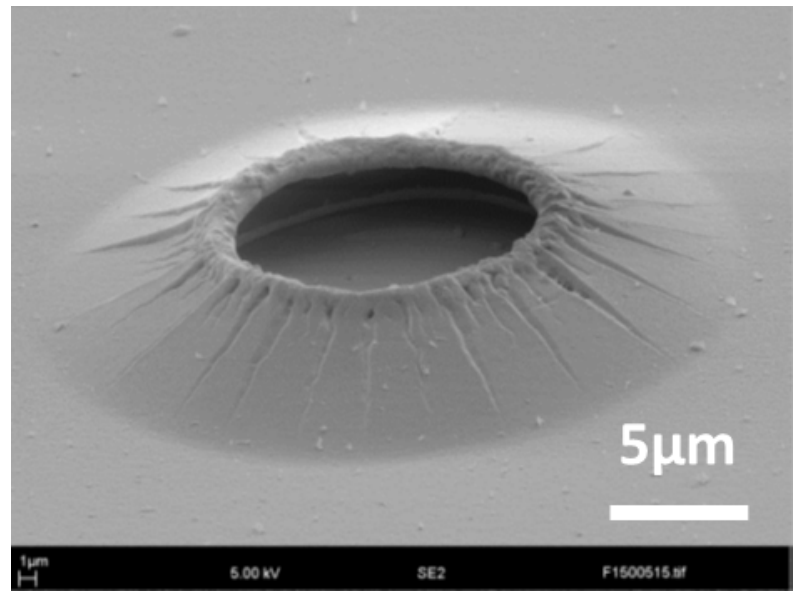

Fig. 6 Organic dielectric layer crater from single pulse SHG 15 ps laser exposure at $6.0 \mathrm{~J} / \mathrm{cm}^{2}$. Crater size $24 \mu \mathrm{m}$ in metal and $17 \mu \mathrm{m}$ in dielectric. Bar denotes $5 \mu \mathrm{m}$.

remove it, which could damage the metal layer.

Figure 7 shows a matrix of single-pulse dielectric layer ablation craters at varying incident pulse energy and z-axis working distance of the focusing lens. All horizontal lines contain craters which received the same laser pulse energy, whilst all vertical line craters were ablated at the same focusing lens working distance. Incident laser energy increases in steps of $650 \mathrm{~nJ}$ in the vertical axis starting from the top line $(9.5 \mu \mathrm{J})$ to the bottom $(15.5 \mu \mathrm{J})$. The beam is defocused from $-225 \mu \mathrm{m}$ away from focal plane (far right) to $-325 \mu \mathrm{m}$ (far left) in z-steps of $10 \mu \mathrm{m}$ outside the focal scheme Rayleigh range. For most of these craters, either no ablation or partial dielectric ablation is observed (broken outline right insert image). For the partially ablated ones, the dielectric residue layers appear mostly solid indicating they have experienced a modest temperature rise and are still attached to the mother layer at the crater edges with occasionally some layer curling observed.

For a narrow range of laser conditions though, selective layer removal of the dielectric layer only, without damage to the metal underlayer is possible (example red solid outlined insert image). The corresponding average fluence range in this case (roughly calculated from a cropped Gaussian propagation algorithm) [20] is around $0.85 \pm 0.06$ $\mathrm{J} / \mathrm{cm}^{2}$. Interestingly, despite this range being 3-4 times higher than the measured metal layer ablation threshold and knowing the dielectric layer is almost totally transparent at $1026 \mathrm{~nm}$, no melting or other mechanical damage to the metal layer is macroscopically observed. This is in line with literature reports on similar metal layer ablation under transparent layer spatial confinement [21]. It should also be noted that tentatively experimenting with the dielectric layer thickness we established that a thinner $(100 \mathrm{~nm})$ dielectric layer can be removed much easier than the standard $500 \mathrm{~nm}$ thick one, but it proved almost impossible to cleanly remove thicker $(800 \mathrm{~nm})$ dielectric layers on the same stack.

One drawback is that the obtained clean via crater size at $45 \mu \mathrm{m}$ diameter is much larger than the targeted via size of $10-15 \mu \mathrm{m}$. Extensive efforts to scale down the size whilst maintaining laser fluence around the optimum found above $0.85 \mathrm{~J} / \mathrm{cm}^{2}$ for selective dielectric ablation, were not successful. It emerged that in order to reduce the via size 

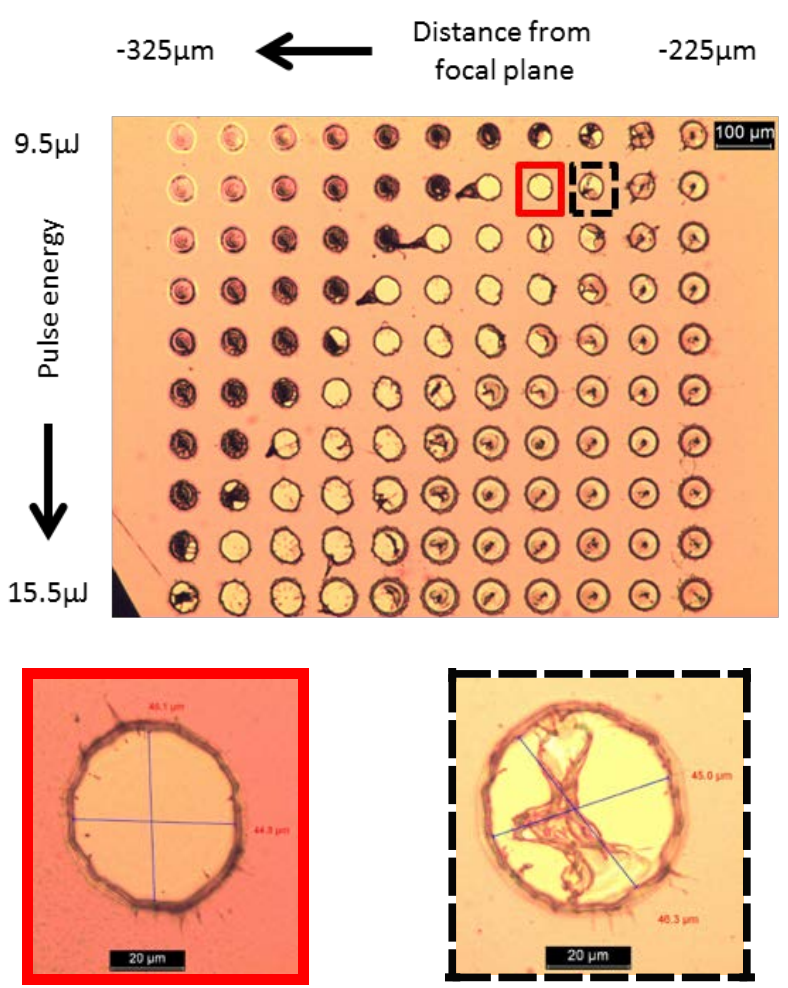

Fig. 7 Optical micrograph matrix of 1026 nm 220 fs single-pulse ablation craters of $500 \mathrm{~nm}$ thick dielectric on $80 \mathrm{~nm}$ thick metal layer at varying distance from the objective focal plane (x-axis) and incident pulse energy (y-axis). An optimum laser ablation process window is observed in a narrow diagonal zone with progressively no via ablation observed towards top left and bottom right part of image. The extreme values in both axes are shown. Inserts show (left) selective dielectric layer removal at $0.85 \mathrm{~J} / \mathrm{cm}^{2}$ versus partial dielectric removal at $0.80 \mathrm{~J} / \mathrm{cm}^{2}$. Insert crater diameter approximately $45 \mu \mathrm{m}$.

and simultaneously achieve selective removal of the organic dielectric transparent layer only, the input laser energy needed to gradually increase. Such spot size dependence to thin film ablation characteristics has been previously reported in metals [22] and dielectrics [23] using nanosecond and ultrashort pulsed lasers, in line with our observations showing that higher incident fluence is required for smaller spots.

Figure 8 shows a typical smaller via crater in the organic dielectric of only $15 \mu \mathrm{m}$ diameter achieved by processing nearer to the lens focal plane with much higher incident fluence of approximately $2.6 \mathrm{~J} / \mathrm{cm}^{2}$. The appearance of the crater is slightly different with more evidence of melting and a noticeable raised round border. Despite such elevated temperature, there is still almost negligible redeposited debris around the crater, the absence of which is very important for manufacturing electronic devices. Although we are not entirely certain about the extent of any dielectric residue or possible metal layer damage at this stage, all electrical connection measurements in tested backplane transistor stacks were successful with almost $100 \%$ yield.

By raising the input energy further whilst processing near focus with a spot size of $20 \mu \mathrm{m}$, both dielectric and metal layer removal is observed at about $3.3 \mathrm{~J} / \mathrm{cm}^{2}$ (Fig. 9). The dielectric crater is larger, exposing the metal layer

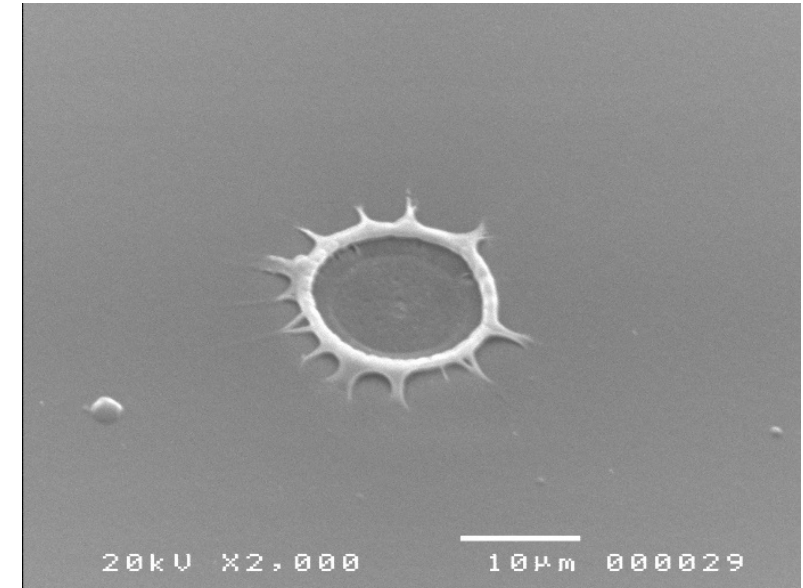

Fig. 8 Single-pulse selective ablation of $500 \mathrm{~nm}$ thick organic dielectric for via interconnection. Metal underlayer is exposed without damage. Laser conditions: $1026 \mathrm{~nm}, 220 \mathrm{fs}, 2.6 \mathrm{~J} / \mathrm{cm}^{2}$, via diameter $15 \mu \mathrm{m}$.

surface for electrical contact as needed. The metal crater is only $10 \mu \mathrm{m}$ in size. In this case, significant quantity of laser debris particles can be seen around the crater indicating the metal amongst other layers experienced a very steep temperature rise. Electrical tests of these via types were also successful with low resistivities of $13 \Omega$ measured upon subsequent layer interconnection trials.

Laser ablation of a transparent dielectric layer on top of an absorbing metal layer represents conditions of spatial confinement and relies on absorption at the interface between the two layers and an associated ejection mechanism. As expected the process is highly dependent on energy input. Critically as the layers are very thin, several different mechanisms are expected to be at play.

The suitability of femtosecond range laser pulse duration to better control the process is not surprising. Despite the relatively high incident laser intensity of $3.8 \times 10^{12}$ $\mathrm{W} / \mathrm{cm}^{2}$ at the optimum operating level (incident laser fluence $0.85 \mathrm{~J} / \mathrm{cm}^{2}$ ) experimental evidence not presented here, deemed this insufficient to induce significant nonlinear absorption, energy deposition and direct ablation of the organic dielectric without the presence of the metal underlayer. Assuming most of the incoming energy after reflection and other losses is deposited on the metal surface

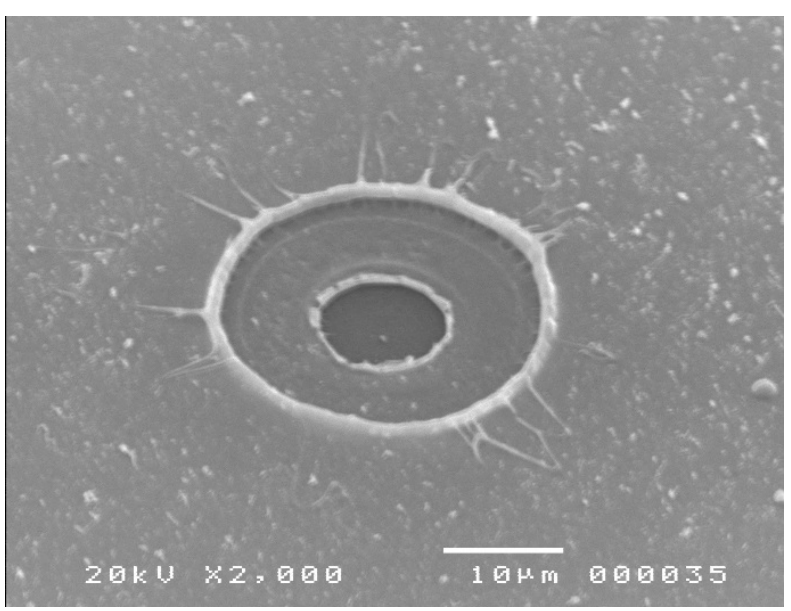

Fig. 9 Single pulse ablation of both $500 \mathrm{~nm}$ thick organic dielectric and $80 \mathrm{~nm}$ metal layers for via interconnection. Laser conditions: $1026 \mathrm{~nm}, 220 \mathrm{fs}, 3.3 \mathrm{~J} / \mathrm{cm}^{2}$. 
therefore, rapid isochoric heating will take place under thermal and stress confinement conditions (e.g. for $80 \mathrm{~nm}$ thick metal, estimated characteristic thermal relaxation time $\approx 0.3-13 \mathrm{ps}$; characteristic stress relaxation time $\approx 4$ 26 ps depending on the exact heated layer depth). In the absence of strain during heating, the thermal stress is expected to exceed $1.43 \mathrm{GPa}$ given a temperature rise of at least $1300 \mathrm{~K}$ similar to above estimates for gold. Without confinement, this should typically lead to metal surface expansion, layer fracture and ejection as described previously in section 3.1. But it is possible [21] that the tensile component of stress in the metal is suppressed by the dielectric overlayer. In such case and depending on the acoustic and thermal contact impedance of the organic dielectric, the relaxation of the initial compressive stress will generate a compressive stress in the dielectric and a weak tension in the metal, insufficient to fracture it. The dielectric layer will experience a large temperature gradient between its "colder" top and "warmer" bottom surfaces and will therefore relax by tensile unloading near the edges. If this exceeds about $70 \mathrm{MPa}$ yield strength, typical for this family of organics, layer fracturing could occur. The remaining input energy is used as kinetic energy for the layer ejection. The significance of this result for via drilling is that the process appears largely blind to the transparent dielectric layer optical properties, and so could be used to remove any such dielectric layer in different OTFT stack configurations, an ideal setup for rapid prototyping technology development.

From plate theory [24], we know that for a given load the maximum deflection of a plate clamped at the edges, scales with the fourth power of its radius and the inverse third power of its thickness. That could explain why higher input energy load is needed for ablation with reducing via size and increasing layer thickness.

A completely different route employing multi-pulse photoincubated [25] UV $355 \mathrm{~nm}$ exposure at 15 ps resulted also in selective removal of the organic dielectric. The crater shown in Fig. 10 was irradiated with 100 million consecutive laser pulses at very low $10 \mathrm{~nJ}$ energy each. The irradiating fluence at $7 \mathrm{~mJ} / \mathrm{cm}^{2}$ is lower than the single

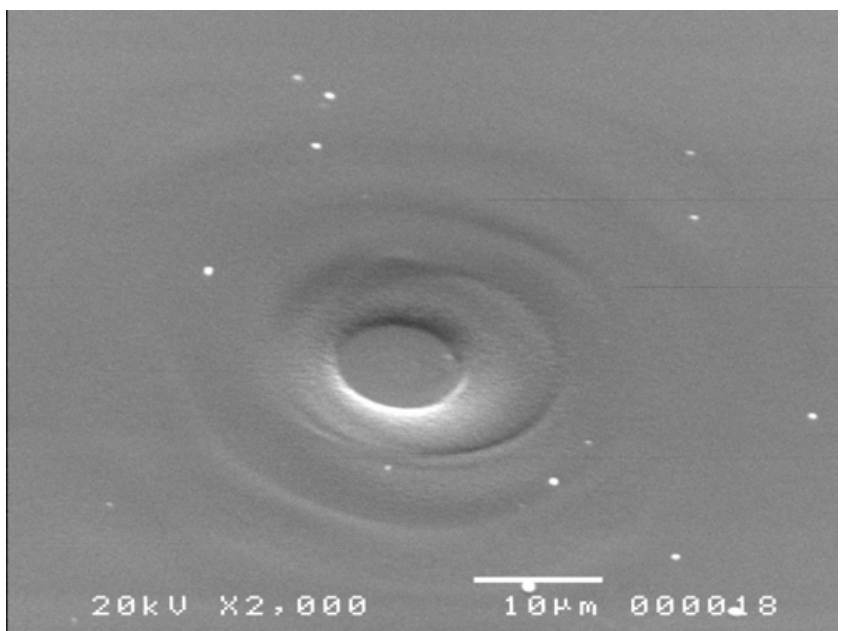

Fig. 10 Via formation by photoincubated controlled ablation of the transparent organic dielectric only. Stop layer: metal. Laser conditions: 355 nm, 15 ps, $10 \mathrm{~nJ}, 100$ million laser pulses exposure. pulse damage threshold of both dielectric and metal (measured for metal as $25 \mathrm{~mJ} / \mathrm{cm}^{2}$ ) but repetitive exposure resulted in controlled ablation of the dielectric layer to stop on the thin metal. In this case, almost certainly gradually increased linear optical absorption permitting laser energy deposition occurs in the dielectric layer from repetitive exposure. This route was not explored further being impractical for our current experimental setup but could be very valuable in future if $\mathrm{MHz}$ ultrafast oscillator sources were to be used.

\section{Conclusions}

We have demonstrated that ultrafast laser S/D transistor patterning and via drilling interconnection for customised fabrication of flexible display OTFT backplanes is possible. Ultrafast lasers offer superior benefits such as a better patterning accuracy, ability to create shortest channel length which enables higher transistor performance and higher process reproducibility comparing to standard printing. This method unlocks the potential for laser direct write for digital fabrication and high value prototyping in this important new technological field.

\section{Acknowledgments}

The research leading to these results has received partial funding from Innovate UK, grant agreement 44069-315222, project SCULPT. DK acknowledges useful discussions with Dr Olivier Uteza, CNRS-LP3, Marseille, France.

\section{References}

[1] K. Suganuma: "Introduction to Printed Electronics", (Springer, New York, 2014), p.14.

[2] B. D. Gates, Q Xu, M. Stewart, D. Ryan, C. Grant Willson, G. M. Whitesides: Chem.Rev., 105, (2005), 1171.

[3] D. Karnakis, M. D. Cooke, Y. F.Chan and S. D. Ogier: Proc. SPIE Laser-based Micro- and Nanopackaging and Assembly VII, San Francisco, (2013) p.86080D.

[4] D. G. Lidzey, M. Voigt, C. Giebeler, A. Buckley, J. Wright, K. Böhlen, J. Fieret and R. Allott: Org. Electron., 6, (2005) 221.

[5] D. Karnakis, A. J. Kearsley and M. Knowles: J. Laser Micro/Nanoengineering, 4, (2009) 218.

[6] R. Mandamparambil, H. Fledderus, G. Van Steenberge and A. Dietzel: Opt.Express, 18, (2010) 7575.

[7] G. Heise, M, Englmaier C. Hellwig, T. Kuznicki, S. Sarrach and H. P. Huber: Appl. Phys. A, 102, (2011) 173.

[8] G. Heise, M.Dickmann, M.Domke, A.Heiss, T. Kuznicki, J.Palm, I. Richter, H. Vogt and H. P. Huber: Appl. Phys. A, 104, (2011) 387.

[9] P. Geys, G. Raciukaitis, E. Miltenis, A. Braun, St.Ragnow: Physics Procedia, 12, (2011) 141

[10] G. Dennler, S. Bereznev, D. Fichou, K. Holl, D. Ilic, R. Koeppe, M. Krebs, A. Labouret, C. Lungenschmied, A. Marchenko, D. Meissner, E. Mellikov, J. Meot, A. Meyer, T. Meyer, H. Neugebauer, A. Opik, N.S. Sariciftci, S. Taillemite, T. Wohrle: Solar Energy, 81 (2007) 947.

[11] S. Xiao, B. Schöps and A. Ostendorf: Physics Procedia, 39, (2012) 594. 
[12] F. Zacharatos, M. Makrygianni, R. Geremia, E. Biver, D. Karnakis, S. Leyder, D. Puerto, P. Delaporte, I. Zergioti: Appl. Surf. Sci., 374, (2016) 117.

[13] see company website for further technical details

[14] D. Yu L. Jiang, F. Wang, X Shi, L. Qu and Y. Lu: Appl. Phys. A, 119, (2015) 1047.

[15] G. Paltauf and P. E. Dyer: Chem.Rev., 103, (2003) 487.

[16] J. P. McDonald, V. R. Mistry, K.E. Ray and S.M. Yalisovel: Appl. Phys. Lett., 88, (2006) 153121.

[17] E. Leveugle, D. S. Ivanov and L. V. Zhigilei: Appl. Phys. A., 79, (2004) 1643.

[18] N. T. Goodfriend, S. V. Starinskiy, O. A. Nerushev, N. M. Bulgakova, A. V. Bulgakov and E. E. B. Campbell: Appl. Phys. A, 122, (2016) 154.

[19] H. Kang, R. Kitsomboonloha, J. Jang and V Subramanian: Adv. Mater., 24, (2012) 3065.
[20] H. Urey, Appl. Opt., 43, (2004) 620.

[21] E. T. Karim, M Shugaev, C. Wu, Z. Lin, R. F. Hainsey and L. V. Zhigilei: J. Appl. Phys., 115, (2014) 183501.

[22] S. Leyder, R. Geremia, C. Moorhouse, D. Karnakis, P. Delaporte, M. Sentis: 7th Int. Symp. on Flexible Organic Electronics (ISFOE14), Thessaloniki (2014)

[23] S. Martin, A. Hertwig, M. Lenzner, J. Kruger, W. Kautek: Appl. Phys. A, 77, (2003) 883.

[24] S. Timoshenko: "Theory of Plates and Shells", (McGraw-Hill Company Inc, New York, 1959) p.56.

[25] P. E. Dyer and D. M. Karnakis: Appl. Phys. A, 59, (1994) 275.

(Received: July 18, 2016, Accepted: October 12, 2016) 